\title{
NON-ISOMORPHISM OF $L_{p}$-SPACES ASSOCIATED WITH FINITE AND INFINITE VON NEUMANN ALGEBRAS
}

\author{
F. A. SUKOCHEV
}

(Communicated by Palle E. T. Jorgensen)

\begin{abstract}
If $\left(M_{1}, \tau_{1}\right)$ is a finite von Neumann algebra and if $\left(M_{2}, \tau_{2}\right)$ is an infinite (semifinite) von Neumann algebra, then $L_{p}\left(M_{1}, \tau_{1}\right)$ and $L_{p}\left(M_{2}, \tau_{2}\right)$
\end{abstract} are non-isomorphic for all $p \in(1, \infty), p \neq 2$.

\section{INTRODUCTION}

The basic question considered here can be formulated as follows. If $E[0, \infty)$ is a rearrangement-invariant space on the positive half line and if $\left(M_{1}, \tau_{1}\right),\left(M_{2}, \tau_{2}\right)$ are semifinite von Neumann algebras, when do the associated symmetric operator spaces $E\left(M_{1}, \tau_{1}\right), E\left(M_{2}, \tau_{2}\right)$ coincide? The source of this question can be found in Banach's book [B] where the non-existence of embeddings of $L_{p}(0,1)$ into $l_{p}$ was exhibited. In the isometric setting, this question appears to be related to the existence of Jordan isomorphisms between $M_{1}$ and $M_{2}$. We mention the paper of Yeadon $[\mathrm{Y}]$ where it is essentially proved that $L_{p}$-spaces associated with semifinite von Neumann algebras are isometric for some $p \in[1, \infty)$ if and only if $M_{1}$ and $M_{2}$ are Jordan isomorphic. Yeadon's result was extended to Lorentz spaces associated with finite algebras in [CMS] where further results about positive isometries between symmetric operator spaces are proved. Finally, the paper of Watanabe [W] considers $*$-preserving isometries for $L_{p}$-spaces associated with arbitrary von Neumann algebras.

The isomorphic formulation of the problem appears to be more difficult. The first results concerning non-commutative algebras are due to C.McCarthy $[\mathrm{M}]$, who considered the question of non-existence of isomorphic embeddings of the Schatten ideals $C_{p}$ into $L_{p}[0,1)$. At least for the case $p>2$, McCarthy showed that $C_{p}$ cannot be finitely represented in $L_{p}[0,1)$. This result expresses the strong difference between the geometry of $L_{p}$-spaces in the commutative and non-commutative settings, for it is easily seen that $C_{p}$ is finitely representable in the non-commutative space $L_{p}(M)$ associated with any von Neumann factor of type $I I_{1}$. Nonetheless, a special case of Theorem 3.1 below asserts that if $p>2$, then $C_{p}$ fails to embed isomorphically into any such non-commutative $L_{p}$-space. In a somewhat different direction, Arazy and Lindenstrauss showed that there exists no isomorphic embedding of $L_{p}[0,1)$ into $C_{p}$, although $L_{p}[0,1)$ is finitely representable in $C_{p}$. This result reflects the fact that $C_{p}$ is an $L_{p}$-space associated with a purely atomic

Received by the editors October 31, 1994.

1991 Mathematics Subject Classification. Primary 46L50; Secondary 47D15, 46E30.

Research supported by the Australian Research Council.

(C)1996 American Mathematical Society 
von Neumann algebra. The theme of the present paper is to compare the structure of $L_{p}$-spaces associated with finite and infinite (semifinite) von Neumann algebras. A principal result (Corollary 3.3) shows that if $1<p<\infty, p \neq 2$, then $L_{p}$-spaces associated with finite von Neumann algebras and those associated with infinite von Neumann algebras are never isomorphic.

The main technical ingredients of our approach are an analogue of the well-known theorem of Kadec-Pelczynski [KP] concerning basic sequences in $L_{p}[0,1), 2<p<$ $\infty$, together with techniques drawn from the theory of rearrangement-invariant spaces. This approach differs quite markedly from those of $[\mathrm{AL}],[\mathrm{M}]$.

\section{Preliminaries}

Let $M$ be a semifinite von Neumann algebra, let $\tau$ be a semifinite normal faithful trace on $M$, let $a=\tau(\mathbf{1})$, where $\mathbf{1}$ is the unit of $M$, and let $K(M, \tau)$ be the $*$-algebra of all $\tau$-measurable operators (see $[\mathrm{FK}]$ ) affiliated with $M$. Let $L_{p}(M, \tau), 1 \leq p<$ $\infty$, be the Banach space of all operators $x \in K(M, \tau)$ such that $\tau\left(|x|^{p}\right)<\infty$ with norm $\|x\|_{p}=\tau\left(|x|^{p}\right)^{1 / p}$, where $|x|=\left(x^{*} x\right)^{1 / 2}$. It is easily verified that $x \in L_{p}(M, \tau)$ if and only if $\mu_{(\cdot)}(x) \in L_{p}[0, a)$ where $\mu_{t}(x)$ for fixed $t \in(0, a]$ is defined by

$$
\mu_{t}(x)=\inf \left\{s \geq 0: \tau\left(\chi_{[s, \infty)}(|x|) \leq t\right\},\right.
$$

where $\chi_{[s, \infty)}(|x|)$ is the spectral projection of $|x|$ corresponding to the interval $(s, \infty)$. If $M$ is $L_{\infty}[0,1)$ (respectively, $\left.l_{\infty}(\mathbf{N})\right)$ and the trace $\tau$ given by integration with respect to Lebesgue measure on the interval $[0,1)$ ( respectively, with respect to counting measure on $\mathbf{N}$ ), then $L_{p}(M, \tau)$ coincides with $L_{p}[0,1)$ (respectively, $l_{p}$ ) and for every $f \in L_{1}[0,1)$ the function $\mu(f)$ coincides with the decreasing rearrangement $f^{*}$ of $|f|$. If $M$ is the von Neumann algebra of all bounded linear operators on $l_{2}$ and $\tau$ is the standard trace, then $L_{p}(M, \tau)$ coincides with the Schatten $p$-class $C_{p}$ of compact operators on the Hilbert space $l_{2}$. The spaces $C_{p}$ are the particular cases of the matrix spaces $C_{E}$ associated with symmetric sequence spaces. If $E$ is a symmetric sequence space, i.e. a Banach space of sequences such that the standard unit vectors form a normalized, 1-symmetric basis of $E$ (see [LT 1]), then we denote by $C_{E}$ the space of compact operators $x$ on $l_{2}$ for which $s(x) \in E$, normed by

$$
\|x\|_{C_{E}}=\|s(x)\|_{E}
$$

where $s(x)=\left\{s_{n}(x)\right\}_{n=1}^{\infty}$ is the sequence of $s$-numbers of $x$, i.e. the eigenvalues of $|x|$ arranged in non-increasing order, counting multiplicity. We shall work with the matrix representation of the elements of $C_{E}$ with respect to some fixed orthonormal basis $\left(e_{i}\right)_{i=1}^{\infty}$ of $l_{2}$. The matrix $x(i, j)$ representing the element $x \in C_{E}$ is defined by $x(i, j)=\left(x e_{j}, e_{i}\right), 1 \leq i, j<\infty$. We shall use the matrix elements $e_{i j} \in C_{E}$ defined by

$$
e_{i j}(k, l)=\delta_{i}^{k} \delta_{j}^{l}, 1 \leq i, j, k, l<\infty .
$$

Recall that a symmetric space $E[0, a)$ is a Banach ideal of locally integrable functions on $[0, a)$ such that if $g$ is a measurable function on $[0, a)$, if $f \in E[0, a)$, and if $g^{*}=f^{*}$, then $g \in E[0, a)$ and $\|f\|_{E[0, a)}=\|g\|_{E[0, a)}$. We define the symmetric space of measurable operators $E(M, \tau)$ associated with $E[0, a)$ and the semifinite von Neumann algebra $(M, \tau), \tau(\mathbf{1})=a \leq \infty$, as follows:

$$
E(M, \tau)=\{x \in K(M, \tau): \mu(x) \in E[0, a)\},\|x\|_{E(M, \tau)}=\|\mu(x)\|_{E[0, a)} .
$$


We shall always assume in this paper that $E[0, a)$ is separable. In this case it is shown in [DDP 1,2], [SC 1,2] that $\left(E(M, \tau),\|\cdot\|_{E(M, \tau)}\right)$ is a Banach space and the norm $\|\cdot\|_{E(M, \tau)}$ is order continuous in the sense $\left\|x_{n}\right\|_{E(M, \tau)} \downarrow 0$ for every sequence $\left\{x_{n}\right\} \subseteq E[0, a)$, such that $0 \leq x_{n} \downarrow 0$.

The measure topology in $K(M, \tau)$ is determined by the fundamental system $\{U(\varepsilon, \delta): \epsilon, \delta>0\}$ of neighbourhoods of zero (see $[\mathrm{FK}]$ ) given by

$$
U(\varepsilon, \delta)=\left\{x \in K(M, \tau):\|x p\|_{M} \leq \varepsilon, \tau(\mathbf{1}-p) \leq \delta \text { for some projection } p \in M\right\},
$$

where $\|\cdot\|_{M}$ is the $C^{*}$-norm on $M$.

We remark that if $M$ is $L_{\infty}[0,1)$ with trace given by integration with respect to Lebesgue measure $m$ on the interval $[0,1)$, then convergence in the measure topology coincides with usual convergence with respect to Lebesgue measure. It should be noted also that $x_{n} \longrightarrow 0$ for the measure topology if and only if $\mu_{t}\left(x_{n}\right) \longrightarrow 0$ for each $t>0$.

The following lemma gives some technical information about convergence in measure in rearrangement-invariant spaces which possess the Fatou property. Recall that $E[0,1)$ has the Fatou property if its unit ball is closed with respect to convergence in measure.

Lemma 1.1. Let E[0,1) be a separable symmetric space with Fatou property, let $M$ be a finite, non-atomic von Neumann algebra with normalised trace $\tau$, and let $\left\{x_{n}\right\}_{n=1}^{\infty} \subseteq E(M, \tau)$ be a bounded sequence. Then there exist a subsequence $\left\{w_{n}\right\}_{n=1}^{\infty} \subseteq\left\{x_{n}\right\}_{n=1}^{\infty}$ and sequences $\left\{y_{n}\right\}_{n=1}^{\infty},\left\{z_{n}\right\}_{n=1}^{\infty} \subseteq E(M, \tau)$ such that $z_{n} \longrightarrow$ 0 for the measure topology, $\mu_{t}\left(y_{n}\right)=\mu_{t}\left(y_{1}\right)$ for all $t \in(0,1]$, and $w_{n}=y_{n}+z_{n}, n \geq$ 1 .

Proof of Lemma 1.1. If $K=\sup _{n}\left\|x_{n}\right\|_{E(M, \mu)}$ and $t>0$, then $\mu_{t}\left(x_{n}\right) \cdot\left\|\chi_{[0, t)}\right\|_{E[0,1)} \leq$ $K$ for all $n \geq 1$. By the Helly Selection Theorem and passing to a subsequence $\left\{w_{n}\right\}_{n=1}^{\infty} \subseteq\left\{x_{n}\right\}_{n=1}^{\infty}$ if necessary, we may assume that there exists a measurable function $f$ on $[0,1)$ such that $\mu_{t}\left(w_{n}\right) \rightarrow f(t)$ for all $t \in(0,1]$. Since $E[0,1)$ has the Fatou property, we can conclude that $f \in E[0,1)$ and $\|f\|_{E[0,1)} \leq K$. Setting $g_{n}=\mu\left(w_{n}\right)-f$, we may assume that $\left\{g_{n}\right\}$ converges to 0 in measure. By Lemma 1.3 of [CKS] (see also [SC 2], Lemma 4.1) for every $n \geq 1$, there exists a rearrangementpreserving isometry $T_{n}: E[0,1) \longrightarrow E(M, \tau)$ such that $T_{n}\left(\mu\left(w_{n}\right)\right)=w_{n}$. Setting $y_{n}=T_{n} f, z_{n}=T_{n}\left(\mu\left(w_{n}\right)-f\right), n \geq 1$, it follows that $w_{n}=y_{n}+z_{n}$ and $\mu_{(\cdot)}\left(y_{n}\right)=$ $f(\cdot)$ for $n \geq 1$. The equality $\mu\left(z_{n}\right)=\mu\left(x_{n}\right)-f, n \geq 1$, implies that $\mu_{t}\left(z_{n}\right) \rightarrow 0$ for all $t>0$, and so $z_{n} \rightarrow 0$ for the measure topology.

Remark 1.2. From the proof of Lemma 1.1 it follows that if $\sup \left\|x_{n}\right\|_{E(M, \tau)} \leq K$ then $\sup \left\|y_{n}\right\|_{E(M, \tau)} \leq K$, and $\sup \left\|z_{n}\right\|_{E(M, \tau)} \leq 2 K$.

Lemma 1.3. If $f_{n}, f \in L_{1}(0,1)$, if $f_{n}=f_{n}^{*}, n \geq 1$, and if $f_{n}(t) \rightarrow f(t)$ a.e. on $(0,1)$, then $m\left(\left\{f_{n} \geq \lambda\right\}\right) \rightarrow m(\{f \geq \lambda\})$ for every $\lambda>0$ such that $m(\{f=\lambda\})=0$.

Proof of Lemma 1.3. By Lemma 1.5 from [CKS] we have

$$
m\left(\left\{f_{n}>\lambda\right\}\right) \rightarrow m(\{f>\lambda\})
$$

for every $\lambda>0$ such that $m(\{f=\lambda\})=0$. Assume that $\liminf m\left(\left\{f_{n}=\lambda\right\}\right)>0$, or without loss of generality, that $m\left(\left\{f_{n}=\lambda\right\}\right) \geq \delta>0$ for all $n=1,2, \ldots$. If

$$
t_{n}=m\left(\left\{f_{n}>\lambda\right\}\right), t_{0}=m(\{f>\lambda\}), n \geq 1,
$$


then $t_{n} \rightarrow t_{0}$ and $f_{n}(t)=\lambda$ for all $t \in\left(t_{n}, t_{n}+\delta\right)$. Hence $f(t)=\lambda$ for all $t \in\left(t_{0}, t_{0}+\delta\right)$, and this contradicts the assumption that $m(\{f=\lambda\})=0$.

If $0<p<\infty$, then $E[0, a)$ is said to be $p$-convex (q-concave) if there exists a constant $C>0$ such that for all finite sequences $\left\{x_{n}\right\}$ in $E[0, a)$

$$
\begin{gathered}
\left\|\left(\sum\left|x_{n}\right|^{p}\right)^{\frac{1}{p}}\right\|_{E[0, a)} \leq C\left(\sum\left\|x_{n}\right\|_{E[0, a)}^{p}\right)^{\frac{1}{p}} \\
\left(C\left\|\left(\sum\left|x_{n}\right|^{q}\right)^{\frac{1}{q}}\right\|_{E[0, a)} \geq\left(\sum\left\|x_{n}\right\|_{E[0, a)}^{q}\right)^{\frac{1}{q}}\right) .
\end{gathered}
$$

The least such constant $C$ is called the $p$-convexity (q-concavity) constant of $E[0, a)$ and is denoted by $M^{(p)}(E[0, a))\left(M_{(q)}(E[0, a))\right)$. It easily can be derived from results in [LT 2] that if the symmetric space $E[0, a)$ is $p$-convex and $q$-concave for some $1<p, q<\infty$, then it is reflexive and has the Fatou property. Recall that a Banach space $E$ is of type 2 if

$$
\int_{0}^{1}\left\|\sum_{1 \leq i \leq n} r_{i}(t) x_{i}\right\| d t \leq A_{E}\left(\sum_{1 \leq i \leq n}\left\|x_{i}\right\|_{E}^{2}\right)^{\frac{1}{2}},
$$

where $\left\{x_{n}\right\}$ is any finite sequence of elements in $E$, the constant $A_{E}$ does not depend on the sequence $\left\{x_{n}\right\}$, and $r_{i}$ denotes the $i$-th Rademacher function for $i \geq 1$. It is established in $[\mathrm{F}]$ that $L_{p}(M, \tau)$ has type 2 for all $2 \leq p<\infty$.

We use standard Banach space terminology and notation throughout, and any term or expression not defined in the text may be found in [LT 1, 2]. For the notation and terminology of the theory of von Neumann algebras, we refer the reader to $[\mathrm{SZ}]$ and $[\mathrm{T}]$. For the basic properties of decreasing rearrangements, we refer to $[\mathrm{KPS}]$ on the commutative setting and to $[\mathrm{FK}]$ in the non-commutative setting.

\section{UnCONDITIONAL BASIC SEQUENCES}

In this section we shall obtain a non-commutative analogue of a result of KadecPelczynski $[\mathrm{KP}]$, which describes unconditional basic sequences in $L_{p}[0,1), p>2$. Throughout this section $M$ is a finite (infinite-dimensional) von Neumann algebra with $\tau(\mathbf{1})=1$. We first show that sequences which converge to zero for the measure topology are approximately disjointly supported. We need first the following simple fact.

Lemma 2.1. Let $E[0,1)$ be a separable symmetric space, and let $x \in E(M, \tau)$. If $\varepsilon>0$ and $e$ is any projection in $M$, then there exists $\delta>0$ such that

$$
\|x e-x f\|_{E(M, \mu)}<\varepsilon
$$

whenever $f \in M$ is a projection with $0 \leq f \leq e$ and $\tau(e-f)<\delta$.

Proof. From the submajorization

$$
\int_{0}^{t} \mu_{s}(x(e-f)) \leq \int_{0}^{t} \mu_{s}(x) \mu_{s}(e-f) d s, \quad t>0,
$$

and the separability of $E[0,1)$, it follows that

$$
\|x(e-f)\|_{E(M, \mu)} \leq\|\mu(x) \mu(e-f)\|_{E(M, \mu)} .
$$

The assertion of Lemma 2.1 now follows immediately from the equality

$$
\mu(e-f)=\chi_{[0, \tau(e-f))} .
$$


Proposition 2.2. Let $E[0,1)$ be separable. If $\left\{x_{n}\right\} \subseteq E(M, \tau)$ and if $x_{n} \longrightarrow 0$ for the measure topology, then there exist two subsequences $\left\{p_{k}\right\},\left\{q_{k}\right\}$ of mutually orthogonal projections and a subsequence $\left\{x_{n(k)}\right\} \subseteq\left\{x_{n}\right\}$ such that

$$
\left\|x_{n(k)}-q_{k} x_{n(k)} p_{k}\right\|_{E(M, \tau)} \rightarrow 0 .
$$

Proof. We suppose first that $0 \leq x_{n}, n \geq 1$. Setting $e_{n}=\chi_{\left[2^{-n}, \infty\right)}\left(x_{n}\right), n \geq 1$, and passing to a subsequence if necessary, we may assume that $\tau\left(e_{n}\right)<2^{-n}, n \geq 1$. It is clear that

$$
\left\|x_{n}-x_{n} e_{n}\right\|_{E(M, \tau)}=\left\|x_{n}\left(\mathbf{1}-e_{n}\right)\right\|_{E(M, \tau)}<2^{-n} \cdot\|\mathbf{1}\|_{E(M, \tau)}, n \geq 1 .
$$

Set

$$
q_{n}=\bigwedge_{m=n+1}^{\infty}\left(\mathbf{1}-e_{m}\right), n=1,2, \ldots,
$$

and observe that $q_{m} \uparrow \mathbf{1}$. Further, for each projection $e \in M$, the parallelogram rule ([SZ], Corollary 4.4) implies that

$$
\tau\left(e-q_{n} \wedge e\right) \leq 1-\tau\left(q_{n}\right), n \geq 1,
$$

so that $q_{n} \wedge e \uparrow_{n} e$ for all projections $e \in M$. It follows inductively from Lemma 2.1 that there exists a strictly increasing sequence $\{n(k)\}_{k=1}^{\infty}$ of natural numbers such that

Setting

$$
\left\|x_{n(k)} e_{n(k)}-x_{n(k)} e_{n(k)} \wedge q_{n(k)}\right\|_{E(M, \tau)}<2^{-k}, k \geq 1 .
$$

$$
p_{k}=e_{n(k)} \wedge q_{n(k)}, k \geq 1,
$$

it is clear that $p_{j} p_{k}=0$ if $j \neq k$ and

$$
\left\|x_{n(k)}-x_{n(k)} p_{k}\right\|_{E(M, \tau)} \rightarrow 0,
$$

as $k \rightarrow \infty$.

Now suppose that $\left\{x_{n}\right\} \in E(M, \tau)$ and that $x_{n} \longrightarrow 0$ for the measure topology. Necessarily, $\left|x_{n}\right| \longrightarrow 0$ for the measure topology, and so there exists a sequence of mutually orthogonal projections $\left\{p_{k}\right\} \subseteq M$ and a subsequence $\left\{x_{n(k)}\right\} \subseteq\left\{x_{n}\right\}$ such that

$$
\left\|\left|x_{n(k)}\right|-\left|x_{n(k)}\right| p_{k}\right\|_{E(M, \tau)} \longrightarrow 0
$$

as $k \rightarrow \infty$. Using the polar decomposition, it follows that

$$
\left\|x_{n(k)}-x_{n(k)} p_{k}\right\|_{E(M, \tau)} \leq\left\|\left|x_{n(k)}\right|-\left|x_{n(k)}\right| p_{k}\right\|_{E(M, \tau)}
$$

for $k=1,2, \ldots$, and so also

$$
\left\|x_{n(k)}-x_{n(k)} p_{k}\right\|_{E(M, \tau)} \longrightarrow 0
$$

as $k \rightarrow \infty$. Now observe also that the sequence $\left\{p_{k} x_{n(k)}^{*}\right\}$ converges to zero for the measure topology. Passing to a further subsequence and relabelling if necessary, it now follows that there exist a subsequence $\left\{x_{n(k)}\right\} \subseteq\left\{x_{n}\right\}$ and a sequence $\left\{q_{k}\right\}$ of mutually orthogonal projections such that

$$
\left\|p_{k} x_{n(k)}^{*}-p_{k} x_{n(k)}^{*} q_{k}\right\|_{E(M, \tau)} \longrightarrow 0
$$

as $k \rightarrow \infty$, and together with (1), this clearly suffices to complete the proof of the Proposition.

Let $x \in K(M, \tau)$. The right and left support projections of $x$ are denoted by $r(x)$ and $l(x)$ respectively . The operators $x, y \in K(M, \tau)$ are said right (respectively, left) disjointly supported if $r(x) r(y)=0$ (respectively, $l(x) l(y)=0)$. 
Proposition 2.3. Let $E[0,1)$ be separable and let $\left\{x_{n}\right\} \subseteq E(M, \tau)$ be a basic sequence. If $\left\{x_{n}\right\}$ is both right and left disjointly supported, then $\left\{x_{n}\right\}$ is isometrically isomorphic to a disjoint basic sequence in $E[0,1)$.

Proof. If $q_{n}=l\left(x_{n}\right), p_{n}=r\left(x_{n}\right)$, then $x_{n}=q_{n} x_{n} p_{n}, n \geq 1$. For each choice of scalars $a_{1}, a_{2}, \ldots, a_{n}, n \geq 1$, we have

$$
\left|\sum_{i=1}^{n} a_{i} x_{i}\right|^{2}=\left(\sum_{i=1}^{n} \bar{a}_{i} p_{i} x_{i}^{*} q_{i}\right)\left(\sum_{i=1}^{n} a_{i} q_{i} x_{i} p_{i}\right)=\sum_{i=1}^{n}\left|a_{i}\right|^{2} p_{i} x_{i}^{*} q_{i} x_{i} p_{i}=\left|\sum_{i=1}^{n} a_{i}\right| x_{i} \|^{2} .
$$

If $\alpha_{1}=0, \alpha_{n}=\sum_{i=1}^{n-1} \tau\left(p_{i}\right), n>1$, and

$$
f_{n}(\cdot)=\mu \cdot-\alpha_{n}\left(x_{n}\right), n \geq 1,
$$

then the sequence $\left\{f_{n}\right\}$ is disjointly supported in $E[0,1)$ and

$$
\left\|\sum_{i=1}^{n} a_{i} x_{i}\right\|_{E(M, \tau)}=\left\|\sum_{i=1}^{n} a_{i}\left|x_{i}\right|\right\|_{E[0,1)}=\left\|\sum_{i=1}^{n} a_{i} f_{i}\right\|_{E[0,1)}
$$

for all $n \geq 1$, and this suffices to complete the proof of the Proposition.

The following Theorem gives a description of unconditional basic sequences in the $L_{p}$-spaces, $p>2$, associated with finite von Neumann algebra M. If $M$ is commutative, then $M$ can be identified with $L^{\infty}(\Omega, \Sigma, \mu)$ where $(\Omega, \Sigma, \mu)$ is a finite measure space. In this case, the result of Theorem 2.4 below is familiar (see [KP] and also [LT 2]).

Following $[\mathrm{KP}]$ for a symmetric space $E(M, \tau)$ and an arbitrary element $x \in$ $E(M, \tau)$ we set

$$
\begin{gathered}
\sigma(x, \varepsilon)=\left\{|x| \geq \varepsilon\|x\|_{E(M, \tau)}\right\}=\chi_{\left[\varepsilon\|x\|_{E(M, \tau)}, \infty\right)}(|x|), \\
\mathbf{M}_{E(M, \tau)}(\varepsilon)=\{x \in E(M, \tau): \tau(\sigma(x, \varepsilon)) \geq \varepsilon\} .
\end{gathered}
$$

It is clear that $x \in \mathbf{M}_{E(M, \tau)}(\varepsilon)$ if and only if $\mu(x) \in \mathbf{M}_{E[0,1)}(\varepsilon)$.

Theorem 2.4. Let $\left\{x_{n}\right\}$ be an unconditional basic sequence in $L_{p}(M, \tau), p>2$, such that $0<\liminf \left\|x_{n}\right\|_{p} \leq \lim \sup \left\|x_{n}\right\|_{p}<\infty$. Either there is a subsequence $\left\{x_{n(k)}\right\}$ which is equivalent to the unit vector basis of $l_{p}$, or $\left\{x_{n}\right\}$ is equivalent to the unit vector basis of $l_{2}$.

Proof. Without loss of generality we can assume that $\left\|x_{n}\right\|_{p}=1$ for all $n \geq 1$. First suppose that for every $\epsilon>0$ there is an index $n_{\varepsilon}$ such that $x_{n_{\varepsilon}}$ does not belong to $\mathbf{M}_{L_{p}(M, \tau)}(\epsilon)$. This means that there exists a subsequence $\left\{\bar{x}_{n}\right\} \subseteq\left\{x_{n}\right\}$ such that $\bar{x}_{n} \rightarrow 0$ in measure. Hence, by Propositions 2.2, 2.3 there is a subsequence $\left\{x_{n(k)}\right\} \subseteq\left\{\bar{x}_{n}\right\}$ which is equivalent to a basic sequence of pairwise disjoint elements of $L_{p}[0,1)$, and so $\left\{x_{n(k)}\right\}$ is equivalent to the unit vector basis of $l_{p}$.

Next, let us assume that there exists $\epsilon>0$ such that $\left\{x_{n}\right\} \subseteq \mathbf{M}_{L_{p}(M, \tau)}(\epsilon)$. Let us show that for any sequence of scalars $\left\{t_{n}\right\}$ the convergence of the series $\sum_{n=1}^{\infty} t_{n} x_{n}$ implies the convergence of the series $\sum_{n=1}^{\infty}\left|t_{n}\right|^{2}$ and conversely. Assume first that the series $\sum_{n=1}^{\infty} t_{n} x_{n}$ unconditionally converges in $L_{p}(M, \tau)$. Since the natural embedding of $L_{p}(M, \tau)$ into $L_{2}(M, \tau)$ is continuous for $p>2$, we have that $\sum_{n=1}^{\infty} t_{n} x_{n}$ 
converges unconditionally in $L_{2}(M, \tau)$, and therefore $\sum_{n=1}^{\infty}\left|t_{n}\right|^{2}\left\|x_{n}\right\|_{2}^{2}<\infty$ (see, for example, [LT 2], 1.e.11). Since $\left\{x_{n}\right\} \in \mathbf{M}_{L_{p}(M, \tau)}(\varepsilon)$, we have

$$
\left\|x_{n}\right\|_{2}=\tau\left(\left|x_{n}\right|^{2}\right)^{1 / 2} \geq \tau\left(\left|x_{n}\right|^{2} \sigma\left(x_{n}, \epsilon\right)\right)^{1 / 2} \geq \epsilon\left\|x_{n}\right\|_{p} \tau\left(\sigma\left(x_{n}, \epsilon\right)\right)^{1 / 2} \geq \epsilon^{3 / 2} .
$$

It follows that $\sum_{n=1}^{\infty}\left|t_{n}\right|^{2}<\infty$. On the other hand, if the series $\sum_{n=1}^{\infty}\left|t_{n}\right|^{2}$ converges, then using the fact that $L_{p}(M, \tau)$ has type 2 , we get for all $m<n$ that

$$
\left\|\sum_{i=m}^{n} t_{i} x_{i}\right\|_{p} \leq K \int_{0}^{1}\left\|\sum_{i=m}^{n} r_{i}(t) t_{i} x_{i}\right\|_{p} d t \leq K A_{p}\left(\sum_{i=m}^{n}\left|t_{i}\right|^{2}\right)^{1 / 2},
$$

where $K$ is the unconditional constant of $\left\{x_{n}\right\}$ and $A_{p}$ is a constant which does not depend on $\left\{x_{n}\right\}$. The last inequality implies the unconditional convergence of $\sum_{n=1}^{\infty} t_{n} x_{n}$ in $L_{p}(M, \tau)$.

Remark 2.5. Suppose that $E[0,1)$ is a symmetric function space which is 2-convex and $q$-concave for some $q<\infty$. It follows from [LT 2], 2.b.3, that $E(M, \tau)$ embeds continuously into $L_{2}(M, \tau)$. By passing to an equivalent symmetric norm if necessary, we may assume that the 2-convexity and $q$-concavity constants of $E[0,1)$ are both equal to 1 (cf. [LT2], 1.d.8). It now follows from [X] and [LT 2], 1.e.16, that $E(M, \tau)$ has type 2 . The same arguments as given in the proof of the preceding Theorem now show that if $\left\{x_{n}\right\} \subseteq \mathbf{M}_{E(M, \tau)}(\varepsilon)$ for some $\varepsilon>0$, then $\left\{x_{n}\right\}$ is equivalent to the unit vector basis of $l_{2}$.

\section{MAin RESUlts}

The following theorem is the principal result of this paper.

Theorem 3.1. Let $F \neq l_{2}$ be a separable symmetric sequence space and let $E[0,1)$ be a separable symmetric function space which is $p$-convex for some $p>2$ and $q$-concave for some $q<\infty$. Then there is no isomorphic embedding of $C_{F}$ into $E(M, \tau)$.

Proof. Without loss of generality we can assume in the sequel that $M$ is a nonatomic von Neumann algebra (see [FK], [SC 2]) and, by Remark 2.5, we may assume also that $M^{(p)}(E[0, a))=M_{(q)}(E[0, a))=1$ and that $E(M, \tau)$ is of type 2 .

Assume that there exists an isomorphic map $T$ from $C_{F}$ onto some subspace of $E(M, \tau)$. Setting $a_{i j}=T e_{i j}, 1 \leq i, j$, it is clear that

$$
0<\left\|T^{-1}\right\|^{-1} \leq\left\|a_{i j}\right\|_{E(M, \tau)} \leq\|T\|<\infty .
$$

Let $n$ be a fixed natural number. Our aim is to show that by deleting appropriate rows and columns and relabelling if necessary, the submatrix $\left[a_{i j}\right]_{i, j=1}^{n}$ admits a decomposition of the form

$$
a_{i j}=b_{i j}+c_{i j}+d_{i j}, \quad 1 \leq i, j \leq n,
$$

where

$$
\mu\left(b_{i j}\right)=\mu\left(b_{i k}\right), \quad 1 \leq i \leq n, 1 \leq j, k \leq n,
$$




$$
\left\|b_{i j}\right\|_{E(M, \tau)} \leq\|T\|,\left\|c_{i j}\right\|_{E(M, \tau)} \leq 2\|T\|,\left\|d_{i j}\right\|_{E(M, \tau)} \leq 4^{-n} n^{-2},
$$

and for every choice of signs $\epsilon_{i}= \pm 1, \eta_{j}= \pm 1$

$$
\left\|\sum_{j=1}^{n} \eta_{j} c_{i j}\right\|_{E(M, \tau)}^{p} \leq n \cdot(2\|T\|)^{p} \text { for all } i=1,2, \ldots, n
$$

$$
\left\|\sum_{i=1}^{n} \epsilon_{i} b_{i j}\right\|_{E(M, \tau)}^{p} \leq n \cdot\|T\|^{p}+4^{-n p} \cdot n^{-p / 2} \text { for all } j=1,2, \ldots, n .
$$

We show first that such a decomposition will lead to a contradiction. We denote by $\sum_{\epsilon_{i}, \eta_{j}}$ the sum over all possible choices of signs $\epsilon_{i}= \pm 1, \eta_{j}= \pm 1$. Using (3), we have

$$
\begin{gathered}
n \cdot 4^{n}=\sum_{\epsilon_{i}, \eta_{j}}\left\|\sum_{i, j=1}^{n} \epsilon_{i} \eta_{j} e_{i j}\right\|_{C_{F}} \leq\left\|T^{-1}\right\| \sum_{\epsilon_{i}, \eta_{j}}\left\|\sum_{i, j=1}^{n} \epsilon_{i} \eta_{j} a_{i j}\right\|_{E(M, \tau)} \\
\leq\left\|T^{-1}\right\| \sum_{\epsilon_{i}, \eta_{j}}\left(\left\|\sum_{i, j=1}^{n} \epsilon_{i} \eta_{j} b_{i j}\right\|_{E(M, \tau)}+\left\|\sum_{i, j=1}^{n} \epsilon_{i} \eta_{j} c_{i j}\right\|_{E(M, \tau)}+\left\|\sum_{i, j=1}^{n} \epsilon_{i} \eta_{j} d_{i j}\right\|_{E(M, \tau)}\right) \\
\leq\left\|T^{-1}\right\| \sum_{\epsilon_{i}, \eta_{j}}\left(\left\|\sum_{i, j=1}^{n} \epsilon_{i} \eta_{j} b_{i j}\right\|_{E(M, \tau)}+\left\|\sum_{i, j=1}^{n} \epsilon_{i} \eta_{j} c_{i j}\right\|_{E(M, \tau)}+4^{-n}\right) .
\end{gathered}
$$

We observe now, using (5) and the fact that $E(M, \tau)$ has type 2, that

$$
\begin{gathered}
\sum_{\epsilon_{i}, \eta_{j}}\left\|\sum_{i, j=1}^{n} \epsilon_{i} \eta_{j} b_{i j}\right\|_{E(M, \tau)}=\sum_{\epsilon_{i}} \sum_{\eta_{j}}\left\|\sum_{j=1}^{n} \eta_{j}\left(\sum_{i=1}^{n} \epsilon_{i} b_{i j}\right)\right\|_{E(M, \tau)} \\
\leq \sum_{\epsilon_{i}} 2^{n} A_{E}\left(\sum_{j=1}^{n}\left\|\sum_{i=1}^{n} \epsilon_{i} b_{i j}\right\|_{E(M, \tau)}^{2}\right)^{\frac{1}{2}} \\
\leq 4^{n} A_{E} n^{\frac{1}{2}}\left(n\|T\|^{p}+4^{-n p} n^{-\frac{p}{2}}\right)^{\frac{1}{p}} \leq A_{E}\left(4^{n} n^{\frac{1}{2}+\frac{1}{p}}\|T\|+1\right) .
\end{gathered}
$$

A similar calculation (using (4)) shows that

$$
\sum_{\epsilon_{i}, \eta_{j}}\left\|\sum_{i, j=1}^{n} \epsilon_{i} \eta_{j} c_{i j}\right\|_{E(M, \tau)} \leq 2 \cdot 4^{n} A_{E} n^{\frac{1}{2}+\frac{1}{p}}\|T\| .
$$

Consequently,

$$
n \cdot 4^{n} \leq\left\|T^{-1}\right\|\left(1+3 \cdot 4^{n} A_{E} n^{\frac{1}{2}+\frac{1}{p}}+A_{E}\right),
$$

and this is a contradiction for $n$ sufficiently large.

We now show the existence of the stated decomposition and establish the estimates $(2)-(5)$. To this end, for simplicity of notation we will denote $\mathbf{M}_{E(M, \tau)}(\varepsilon)$ by $\mathbf{M}(\varepsilon)$ and set

$$
\varepsilon_{i j}=\sup \left\{\varepsilon: a_{i j} \in \mathbf{M}(\varepsilon)\right\},
$$


for all $i, j \geq 1$. Observe that $\liminf _{j \rightarrow \infty} \varepsilon_{i j}>0$. In fact, if it is not the case for some $i \geq 1$, then it follows from Propositions 2.2 and 2.3 that there exist a subsequence $\left\{a_{i j(k)}\right\}_{k=1}^{\infty} \subseteq\left\{a_{i j}\right\}_{j=1}^{\infty}$ and a right and left pairwise disjointly supported sequence $\left\{b_{k}\right\} \subseteq E(M, \tau)$ which is isometrically equivalent to a disjointly supported basic sequence in $E[0,1)$ such that $\left\|b_{k}-a_{i j(k)}\right\|_{E(M, \tau)} \rightarrow 0$ as $k \rightarrow \infty$. The $p$-convexity of $E[0,1)$ implies that

$$
\left\|\sum_{1 \leq k \leq m} b_{k}\right\|_{E(M, \tau)} \leq\left(\sum_{1 \leq k \leq m}\left\|b_{k}\right\|_{E(M, \tau)}^{p}\right)^{\frac{1}{p}}
$$

for all $m \geq 1$. This, however, contradicts the fact that the sequence $\left\{a_{i j(k)}\right\}_{k=1}^{\infty}$ is equivalent to the unit vector basis of $l_{2}$. It follows from a simple inductive argument that for each $i \geq 1$, there exists a strictly increasing sequence $\left\{j^{(i)}(k)\right\}_{k \geq i}$ of natural numbers such that $\left\{j^{(i+1)}(k)\right\}_{k \geq i+1} \subseteq\left\{j^{(i)}(k)\right\}_{k \geq i+1}, 0<\varepsilon_{i}=\lim _{k \rightarrow \infty} \varepsilon_{i j(i)}(k)$ exists, and $a_{i j^{(i)}(k)} \notin \mathbf{M}\left(2 \varepsilon_{i}\right)$, for all $k \geq i$. By suitable relabelling, we may assume that $j^{(i)}(k)=k, k \geq i \geq 1$. We show that $\lim _{i \rightarrow \infty} \varepsilon_{i}=0$. If this is not the case, then we may assume that there exists $\varepsilon>0$ such that $\varepsilon_{i}>\varepsilon$ for all $i \geq 1$. Consequently, there exists a strictly increasing sequence $\{j(i)\}_{i=1}^{\infty}$ such that $\left\{a_{i j(i)}\right\}_{i=1}^{\infty} \subseteq \mathbf{M}\left(\frac{\varepsilon}{2}\right)$. It follows from Remark 2.5 that the sequence $\left\{a_{i j(i)}\right\}$ is equivalent to the unit vector basis of $l_{2}$. However, as the sequence $\left\{e_{i j(i)}\right\} \subseteq C_{F}$ is left and right disjointly supported, it follows that the sequence $\left\{a_{i j(i)}\right\}_{i=1}^{\infty}$ is equivalent to the unit vector basis of $F$ and this contradicts the assumption that $F$ is not isomorphic to $l_{2}$.

Using Lemma 1.1, Remark 1.2, Propositions 2.2, 2.3 and appropriate relabelling, a simple inductive argument shows that for all $i \geq 1$, there exist functions $b_{i}(t) \in$ $E[0,1)$ and sequences $\left\{b_{i j}\right\}_{j \geq i},\left\{c_{i j}\right\}_{j \geq i},\left\{d_{i j}\right\}_{j \geq i}$ with

$$
a_{i j}=b_{i j}+c_{i j}+d_{i j}, \quad \text { for all } i \leq j
$$

such that $\left\{c_{i j}\right\}_{j \geq i}$ is isometrically equivalent to a disjointly supported sequence in $E[0,1), \mu\left(b_{i j}\right)=b_{i}$ for all $j \geq i$, and $\mu_{t}\left(a_{i j}\right) \rightarrow b_{i}(t)$ a.e. on $(0,1)$, and for which

$$
\left\|b_{i j}\right\|_{E(M, \tau)} \leq\|T\|,\left\|c_{i j}\right\|_{E(M, \tau)} \leq 2\|T\|,\left\|d_{i j}\right\|_{E(M, \tau)} \leq 4^{-n} n^{-2}, j \geq i .
$$

Since $E[0,1)$ is $p$-convex, it follows that

$$
\left\|\sum_{j=k}^{m} \eta_{j} c_{i j}\right\|_{E(M \tau)}^{p} \leq(m-k)(2\|T\|)^{p} \quad \text { for all } k \leq j \leq m ; k, m \geq i ; i \geq 1
$$

and every choice of signs $\eta_{j}= \pm 1$.

We show now that the sequence $\left\{b_{k}\right\}$ contains a subsequence which converges to zero for the measure topology. It is clear that we may assume that $\left\|b_{k}\right\|_{E[0,1)} \geq C>$ 0 for all $k=1,2, \ldots$. This implies that for every $\varepsilon>0$, there is $k(\epsilon)$ such that $b_{k(\varepsilon)} \notin$ $\mathbf{M}_{E[0,1)}(\varepsilon)$. Indeed, suppose that $\varepsilon>0$ and that $\left\{b_{k}\right\}_{k=1}^{\infty} \subseteq \mathbf{M}_{E[0,1)}\left(\epsilon\left(C\left\|T^{-1}\right\|\right)^{-1}\right)$. For all $k \geq 1$, choose $\delta_{k}>0$ such that $0<\varepsilon-\delta_{k}<2^{-1} \varepsilon$ and $m\left(\left\{t: b_{k}(t)=\right.\right.$ $\left.\left.\delta_{k}\left\|T^{-1}\right\|^{-1}\right\}\right)=0$ and using the fact that $\mu\left(a_{k j}\right) \rightarrow \mu\left(b_{k}\right)$ a.e. on $(0,1)$, it follows from Lemma 1.3 that

$$
m\left(\left\{t: \mu_{t}\left(a_{k j}\right) \geq \delta_{k}\left\|T^{-1}\right\|^{-1}\right\}\right) \rightarrow m\left(\left\{t: b_{k}(t) \geq \delta_{k}\left\|T^{-1}\right\|^{-1}\right\}\right) .
$$


So, for sufficiently large $j$, it follows that

$$
\begin{gathered}
m\left(\left\{t: \mu_{t}\left(a_{k j}\right) \geq \varepsilon\left(2\left\|T^{-1}\right\|\right)^{-1}\right\}\right) \geq m\left(\left\{t: \mu_{t}\left(a_{k j}\right) \geq \delta_{k}\left(\left\|T^{-1}\right\|\right)^{-1}\right\}\right) \\
\geq m\left(\left\{t: b_{k}(t) \geq \delta_{k}\left(\left\|T^{-1}\right\|\right)^{-1}\right\}\right)-\varepsilon\left(2 C\left\|T^{-1}\right\|\right)^{-1} \\
\geq m\left(\left\{t: b_{k}(t) \geq \varepsilon\left\|b_{k}\right\|_{E[0,1)}\left(C\left\|T^{-1}\right\|\right)^{-1}\right\}\right)-\varepsilon\left(2 C\left\|T^{-1}\right\|\right)^{-1} \geq \varepsilon\left(2 C\left\|T^{-1}\right\|\right)^{-1} .
\end{gathered}
$$

Since $\left\|a_{k j}\right\|_{E(M, \tau)} \leq\|T\|<\infty$, it follows that $a_{k j} \in \mathbf{M}(\delta)$ for each $k$ and sufficiently large $j$ where

$$
\delta=\min \left(\varepsilon\left(2 C\left\|T^{-1}\right\|\right)^{-1}, \varepsilon\left(2\|T\| \cdot\left\|T^{-1}\right\|\right)^{-1}\right) .
$$

This however contradicts the fact that $\varepsilon_{k} \rightarrow 0$ and that $a_{k j} \notin \mathbf{M}\left(2 \varepsilon_{k}\right)$. It now follows that there exists a subsequence $\left\{b_{i(k)}\right\} \subseteq\left\{b_{k}\right\}$ which converges to zero for the measure topology. Using Proposition 2.2 applied in the space $E[0,1)$, we may assume that

$$
\left\|\sum_{i=1}^{n} b_{i(k)}\right\|_{p}^{p} \leq n \cdot\|T\|^{p}+4^{-n p} \cdot n^{-p / 2}
$$

Consequently for every choice of signs $\epsilon_{k}= \pm 1$ and every $j \geq i(k), k=1,2, \ldots$, it follows that

$$
\begin{gathered}
\left\|\sum_{k=1}^{n} \epsilon_{k} b_{i(k) j}\right\|_{E(M, \tau)}^{p}=\left\|\mu\left(\sum_{k=1}^{n} \epsilon_{k} b_{i(k) j}\right)\right\|_{E 0,1)}^{p} \leq\left\|\sum_{k=1}^{n} \mu\left(\epsilon_{k} b_{i(k) j}\right)\right\|_{E[0,1)}^{p}= \\
\left\|\sum_{k=1}^{n} \mu\left(b_{i(k) j}\right)\right\|_{E[0,1)}^{p}=\left\|\sum_{k=1}^{n} b_{i(k)}\right\|_{E[0,1)}^{p} \leq n \cdot\|T\|^{p}+4^{-n p} n^{-p / 2} .
\end{gathered}
$$

It now follows that the stated decomposition and the estimates $(2)-(5)$ hold for the $n \times n$ submatrix $\left[a_{i(k) j}\right], 1 \leq k \leq n, n \leq j \leq 2 n-1$, and all that now remains is a simple change of notation. This completes the proof of the Theorem.

Corollary 3.2. Let $M_{1}$ be a finite von Neumann algebra with finite, normal, faithful trace $\tau_{1}$, and let $M_{2}$ be an infinite von Neumann algebra with semifinite, normal, faithful trace $\tau_{2}$. If $E(0, \infty)$ is a rearrangement invariant space which is $p$-convex and $q$-concave for some $p, q \in(1, \infty)$ with either $p>2$ or $q<2$, then $E\left(M_{1}, \tau_{1}\right)$ and $E\left(M_{2}, \tau_{2}\right)$ are non-isomorphic.

Proof. Using Corollary 5.16 of [DDP 2] and 1.d.4 of [LT 2] we need consider only the case $E(0, \infty)$ is $p$-convex for some $p>2$. From the well-known structure theory of infinite (semi-finite) von Neumann algebras (see [T], Chapter V) it is not difficult to find a subspace $G$ of $E\left(M_{2}, \tau_{2}\right)$ which is isometrically isomorphic to the space $C_{F}$, where $F$ is the symmetric sequence space corresponding to the subspace of $E[0, \infty)$ generated by the elements $\left\{\chi_{[n \delta,(n+1) \delta)}\right\}_{n=0}^{\infty}$, for some $\delta>0$. From our assumptions it follows that $F$ is not isomorphic to $l_{2}$, and the Corollary now follows immediately.

Corollary 3.3. Let $M_{1}$ be a finite von Neumann algebra with finite, normal, faithful trace $\tau_{1}$, and let $M_{2}$ be an infinite von Neumann algebra with semifinite, normal, faithful trace $\tau_{2}$. Then $L_{p}\left(M_{1}, \tau_{1}\right)$ and $L_{p}\left(M_{2}, \tau_{2}\right)$ are non-isomorphic for all $p \in(1, \infty), p \neq 2$.

Remark 3.4. Finally we remark that the von Neumann algebras $L_{\infty}, l_{\infty}$ and $B(H)$ are examples of von Neumann algebras of type $I$. The methods that are presented 
here are the important ingredients needed to complete the isomorphic classification of all possible types of $L_{p}$-spaces associated with algebras of such type. This classification was announced in [SC 1]. There are nine possible isomorphic types of $L_{p}$-spaces associated with the algebras of type $I$ listed. Details of proof will be published elsewhere.

\section{ACKNOWLEDGEMENT}

The author wishes to thank P.G.Dodds for his helpful comments on earlier versions of the present paper.

\section{REFERENCES}

[AL] J. Arazy and J. Lindenstrauss, Some linear topological properties of the spaces $C_{p}$ of operators on Hilbert spaces, Compositio Math. 30 (1975), 81-111. MR 51:8876

[B] S. Banach, Théorie des opérations linéaires, Warszawa, 1932.

[CMS] V. I. Chilin, A. M. Medzitov, and F. A. Sukochev, Isometries of non-commutative Lorentz spaces, Math. Zeit. 200 (1989), 527-545. MR 90i:46113

[CKS] V. I. Chilin, A. V. Krygin, and F. A. Sukochev, Local uniform and uniform convexity of non-commutative symmetric spaces of measurable operators, Math. Proc. Camb. Phil. Soc. 111 (1992), 355-368. MR 93b:46124

[DDP1] P. G. Dodds, T. K. Dodds, and B. de Pagter, Non-commutative Banach function spaces, Math. Zeit. 201 (1989), 583-597. MR 90j:46054

[DDP2] _ Noncommutative Köthe duality, Trans. Amer. Math. Soc. 339 (1993), 717-750. MR 94a:46093

[F] T. Fack, Type and cotype inequality for non-commutative $L^{p}$-spaces, J. Operator Theory 17 (1987), 255-279. MR 88g:46069

[FK] T. Fack and H. Kosaki, Generalized s-numbers of $\tau$-measurable operators, Pacific J. Math. 123 (1986), 269-300. MR 87h:46122

[KP] M. I. Kadec and A. Pelczynski, Bases, lacunary sequences and complemented subspaces in the spaces $L_{p}$, Studia Math. 21 (1962), 161-176. MR 27:2851

[KPS] S. G. Krein, Ju. I. Petunin, and E. M. Semenov, Interpolation of linear operators, Translations of Mathematical Monographs, Amer. Math. Soc., Providence, R.I., 1982. MR 84j: 46103

[LT 1] J. Lindenstrauss and L. Tzafriri, Classical Banach spaces I. Sequence spaces, SpringerVerlag, New-York, 1977. MR 58:17766

[LT 2] Classical Banach spaces II. Function spaces, Springer-Verlag, New-York, 1979.

[M] C. McCarthy, $C_{p}$, Israel J. Math. 5 (1967), 249-271. MR 37:735

[SC 1] F. A. Sukochev and V. I. Chilin, Symmetric spaces on semifinite von Neumann algebras, Soviet Math. Dokl. 42 (1992), 97-101.

[SC 2] Weak convergence in non-commutative symmetric spaces, J. Operator Theory 31 (1994), 35-65. CMP 95:08

[SZ] S. Stratila and L. Zsido, Lectures on von Neumann algebras, Abacus Press, England, 1975. MR 81j:46089

[T] M. Takesaki, Theory of operator algebras I, Springer-Verlag, New York, 1979. MR 81e: 46038

[W] K. Watanabe, On isometries between non-commutative $L^{p}$-spaces associated with arbitrary von Neumann algebras, J. Operator Theory 28 (1992), 267-279. MR 95b:46093

[X] Xu, Convexité uniforme des espaces symmétriques d'opérateurs mesurables, C. R. Acad. Sci. Paris. Serie I 309 (1989), 251-254.

[Y] F. Yeadon, Isometries of non-commutative $L_{p}$-spaces, Math. Proc. Cambridge Philos. Soc. 90 (1981), 41-50. MR 82g:46108

Department of Mathematics and Statistics, School of Information Science and Technology, The Flinders University of South Australia, GPO Box 2100, Adelaide, SA 5001, Australia

E-mail address: sukochev@ist.flinders.edu.au 